\title{
Algèbre et géométrie, 1956-1994
}

\author{
Jean-Pierre Serre
}

\section{(2) OpenEdition}

Journals

Édition électronique

URL : https://journals.openedition.org/annuaire-cdf/1079

DOI : 10.4000/annuaire-cdf.1079

ISBN : 978-2-7226-0325-7

ISSN : 2109-9227

Éditeur

Collège de France

Édition imprimée

Date de publication : 1 avril 2013

Pagination : 807-808

ISBN : 978-2-7226-0198-7

ISSN : 0069-5580

Référence électronique

Jean-Pierre Serre, «Algèbre et géométrie, 1956-1994 », L'annuaire du Collège de France [En ligne], 112 |

2013, mis en ligne le 22 novembre 2013, consulté le 22 août 2022. URL : http://

journals.openedition.org/annuaire-cdf/1079 ; DOI : https://doi.org/10.4000/annuaire-cdf.1079 
« Société et cultures, le point de vue des historiens (1960-2000) », École doctorale (Paris VII, Société et histoire moderne), 16 novembre 2011.

Conférence d'ouverture «Les conditions de l'expérience», au colloque Mobilité et circulation des savoirs, Université de Paris VII, 17 novembre 2011.

«Cheval et politique, transmission et éducation», conférence à l'université de Genève, 22 novembre 2011.

«Équitation et société », Centre d'histoire de la Renaissance (P. Brioist), université de Tours, 11 février 2012.

«Le Baroque, culture et réflexion sur l'âge baroque, $\mathrm{XVII}$-XVIII ${ }^{\mathrm{e}}$ siècles », conférence à l'Istituto San Paulo - 1563, 7 mars 2012.

« Histoire des idées, histoire culturelle, histoire des sociabilités », intervention au séminaire de doctorat, M. Roggero, université de Turin, 8 mars 2012.

«L'équitation et la politique (XVI $-\mathrm{XIX}^{\mathrm{e}}$ siècles) », communication à la Société des lettres, sciences et arts de Rodez, 5 mai 2012.

\section{FONCTIONS ADMINISTRATIVES}

Directeur de la Revue d'histoire moderne et contemporaine.

Membre du conseil scientifique de l'École des Chartes.

Membre du conseil scientifique de l'université de Paris I.

\section{DISTINCTION}

Élu membre académicien correspondant étranger de la classe des sciences morales, section des sciences philosophiques, pédagogiques et morales de l'Institut de Bologne (juillet 2011).

\section{Jean-Pierre SERRE, membre de l'Institut (Académie des sciences)}

\section{Algèbre et géométrie, 1956-1994}

\section{Publications}

Serre J.-P., Le groupe de Cremona et ses sous-groupes finis, séminaire Bourbaki 2008/2009, exposé no 1000, Astérisque, 332, 2010, 75-100.

Serre J.-P., lettre à N. Karoubi, reproduite en appendice dans : Karoubi N., Clifford modules and invariants of quadratic forms, Journal of K-theory, 9, 2012, 1-44.

Serre J.-P., Lectures on $N \times(p)$, AK Peters, CRC Press, Taylor and Francis, New York, 2012, $174 \mathrm{p}$.

Serre J.-P. et Nicolas J.-L., Formes modulaires modulo 2 : l'ordre de nilpotence des opérateurs de Hecke, C.R.A.S., 350, 2012, 343-348. 
Serre J.-P. et Nicolas J.-L., Formes modulaires modulo 2 : structure de l'algèbre de Hecke, C.R.A.S., 350, 2012, 449-454.

\section{EXPOSÉS}

Introduction to the Sato-Tate conjecture, Oberwolfach, juillet 2011 ; Montréal, septembre 2011 ; Harvard, octobre 2011.

Variation with $p$ of the number of solutions mod $p$ of a system of polynomial equations, Harvard, septembre 2011 ; Montréal, septembre 2011 ; Munich, novembre 2011.

Integration and equidistribution, Harvard, octobre 2011.

The action of Hecke operators on the modular forms mod 2 in level 1, Montréal, octobre 2011 ; Harvard, octobre 2011.

Groupes de Galois: le cas abélien, Colloque du bicentenaire d'Evariste Galois, Paris, octobre 2011.

Discriminants, EPFL, Lausanne, novembre 2011.

Formes modulaires modulo 2 de niveau 1, Orsay, décembre 2011 ; Caen, mai 2012.

Principe de Hasse pour les G-formes traces, d'après Bayer-Parimala, Lausanne, EPFL, mai 2012 ; Paris, ENS, juin 2012.

Quelques applications des groupes algébriques linéaires, Bures sur Yvette, IHES, juin 2012.

Revêtements des courbes algébriques, Paris, IHP, juin 2012.

\section{DISTINCTION}

Élu membre honoraire de la Société mathématique d’Espagne, novembre 2011.

\section{Pierre TOUBeRt, membre de l'Institut (Académie des inscriptions et belles-lettres) \\ Occident méditerranéen au Moyen Âge, 1992-2003}

\section{Publications}

Toubert P. et Sénac Ph. (dir. et collab.), Villa 3 - Histoire et archéologie des sociétés de la vallée de l'Ebre (VII ${ }^{e}-\mathrm{XI}^{e}$ siècles), coll. «Méridiennes », série «Études médiévales ibériques », Presses de l'université de Toulouse-Le Mirail, Toulouse, 2010.

Toubert P. et Sénac Ph. (dir. et éd.), Villa 4 - Histoire et archéologie de l'Occident musulman (VII $-\mathrm{XI}^{e}$ siècles) Al-Andalus, Maghreb, Sicile, collection "Méridiennes ", série «Études médiévales ibériques », Presses de l'université de Toulouse-Le Mirail, Toulouse, 2012, 285 p. 